\title{
Acute and sustained effects of aerosolized vs. bolus surfactant therapy in premature lambs with respiratory distress syndrome
}

\author{
Carmen Rey-Santano', Victoria E. Mielgo', Leire Andres², Estibaliz Ruiz-del-Yerro' ${ }^{1}$, Adolfo Valls-i-Soler ${ }^{1,3}$ and Xabier Murgia'
}

\begin{abstract}
BACKGROUND: Surfactant (SF) instillation may produce acute deleterious effects on gas exchange and both systemic and cerebral hemodynamics. Our aim was to compare the effects of aerosolized SF (SF-aero) with those of bolus SF (SF-bolus) administration on gas exchange, lung mechanics, and cardiovascular function in premature lambs with respiratory distress syndrome (RDS).
\end{abstract}

METHODS: Fourteen preterm lambs (85\% gestation) were randomly assigned to receive SF-aero or SF-bolus. Oxygenation index (Ol), $\mathrm{PaCO}_{2}$, cardiovascular parameters, carotid blood flow (CBF), lung compliance (mean dynamic compliance), and tidal volume $\left(V_{T}\right)$ were measured every $30 \mathrm{~min}$ for $6 \mathrm{~h}$. Biochemical and histological analyses were performed.

RESULTS: After delivery, lambs developed severe RDS (inspiratory fraction of oxygen: $1 ; \mathrm{pH}<7.15 ; \mathrm{PaCO}_{2}>80 \mathrm{~mm} \mathrm{Hg} ; \mathrm{PaO}_{2}<$ $30 \mathrm{~mm} \mathrm{Hg}$, mean dynamic compliance $<0.08 \mathrm{ml} / \mathrm{cm} \mathrm{H}_{2} \mathrm{O} / \mathrm{kg}$ ). By $60 \mathrm{~min}$ after treatment, both groups showed an improvement in $\mathrm{OI}, \mathrm{PaCO}_{2^{\prime}}$ mean dynamic compliance, and $V_{\mathrm{T}}$ that was maintained until the end of the experiment. $\mathrm{PaCO}_{2}$ and $\mathrm{CBF}$ increased significantly in the SF-bolus group during the first 15-30 min, without concomitant changes in cardiovascular parameters, whereas in the SF-aero group, $\mathrm{PaCO}_{2}$ and $\mathrm{CBF}$ decreased gradually. SF-aero induced less alveolar hemorrhage and inflammation.

CONCLUSION: SF-aero produced improvements in gas exchange and lung mechanics similar to those produced by bolus administration but with less lung injury and fewer cerebral hemodynamic changes.

$\mathbf{N}$ eonatal respiratory distress syndrome (RDS) is caused primarily by a deficiency in pulmonary surfactant (SF). The current clinical treatment of infants with RDS includes endotracheal intubation and rapid intratracheal instillation of exogenous SF. Although this treatment reduces morbidity and mortality, intratracheal instillation of SF has been associated with adverse effects such as transient hypoxia, desaturation, hypercapnia, and hemodynamic imbalance $(1,2)$, which might in turn cause fluctuations in cerebral blood flow and electroencephalogram abnormalities $(1,2)$.
Administering SF more slowly, in smaller volumes or using less viscous SFs, might theoretically prevent the "peridosing" adverse events observed during SF administration by reducing endotracheal tube obstructions and by producing smaller or more gradual changes in gas exchange and lung function, as well as less acute changes in systemic and cerebral hemodynamics (3). However, an inadequate instillation of SF (slow tracheal infusion or multiple small doses) may increase the rate of "poor responders" to SF treatment because the distribution of SF within the lungs may be less homogeneous $(4,5)$.

Delivery of aerosolized SF (SF-aero) might be a useful alternative to tracheal instillation for the treatment of RDS, but experimental and clinical results with this technique remain inconsistent (6-13). Discrepancies in the results can be attributed to the use of different animal models of lung injury (neonatal RDS vs. adult RDS), aerosol devices (jet vs. ultrasonic), sites for the placement of the aerosol devices (endotracheal tube vs. nasal prong), and SF preparations (natural vs. synthetic), as well as variations in the doses used. Furthermore, published studies have mainly focused on pulmonary efficacy, but limited data are available on systemic and cerebral hemodynamics.

Therefore, this study was designed to determine the pulmonary efficacy of delivery of SF-aero in comparison with the standard method, namely, bolus instillation, and also to compare the systemic and cerebral hemodynamic patterns observed with the two administration methods. We hypothesized that delivering SF as an aerosol might partly avoid the acute peridosing adverse events reported during rapid intratracheal instillation while still achieving a significant improvement in lung function in preterm lambs with severe RDS.

\section{RESULTS}

Briefly, ewes had adequate and stable gas exchange (pH: $7.42 \pm$ 0.06; $\mathrm{PaCO}_{2}: 40 \pm 9 \mathrm{~mm} \mathrm{Hg} ; \mathrm{PaO}_{2}: 130 \pm 32 \mathrm{~mm} \mathrm{Hg}$ ) and mean arterial blood pressure (MABP) of $70 \pm 10 \mathrm{~mm} \mathrm{Hg}$ throughout the study. 


\section{Pulmonary Gas Exchange}

Fetal $\mathrm{PaO}_{2}$ values were similar in the bolus SF (SF-bolus) and SF-aero groups ( $25 \pm 5$ vs. $26 \pm 4 \mathrm{~mm} \mathrm{Hg}$, not significant (NS)), as were baseline $\mathrm{PaO}_{2}$ levels after stabilization on mechanical ventilation ( $17 \pm 12$ vs. $22 \pm 14 \mathrm{~mm} \mathrm{Hg}$, NS). In the SF-bolus group, there was a rapid increase in $\mathrm{PaO}_{2} 15 \mathrm{~min}$ after tracheal SF instillation to a level that was significantly higher than that in the SF-aero group ( $91 \pm 27$ vs. $35 \pm 13 \mathrm{~mm}$ $\mathrm{Hg}$ ). However, by $30 \mathrm{~min}$ after SF administration, $\mathrm{PaO}_{2} \mathrm{lev}$ els were similar in the two groups and remained stable (with similar inspiratory fraction of oxygen $\left(\mathrm{FiO}_{2}\right)$ ) until the end of experiment; no significant differences were found between the groups.

The oxygenation index (OI) fell during the first $60 \mathrm{~min}$ in both groups: from $80 \pm 30$ to $15 \pm 9$ after SF-bolus instillation and from $70 \pm 25$ to $14 \pm 6$ after SF aerosolization. During the acute period after SF therapy, however, the OI decreased more slowly in the SF-aero than the SF-bolus group. Subsequently, values in the two groups were similar, remaining stable near the values reached $60 \mathrm{~min}$ after SF delivery (Figure 1a). At baseline, the ventilatory efficiency index was similar in the two groups $(0.03 \pm 0)$, but $5 \mathrm{~min}$ after SF aerosolization, the ventilatory efficiency index increased significantly in the SF-aero group as compared with the SF-bolus group. Then, however, the values followed a similar trend, increasing for $2 \mathrm{~h}$ (SF-bolus: $0.15 \pm 0.05$ and SF-aero: $0.20 \pm 0.08$ ), and thereafter the two groups maintained values that were not significantly different until the end of the experiment (Figure 1b).
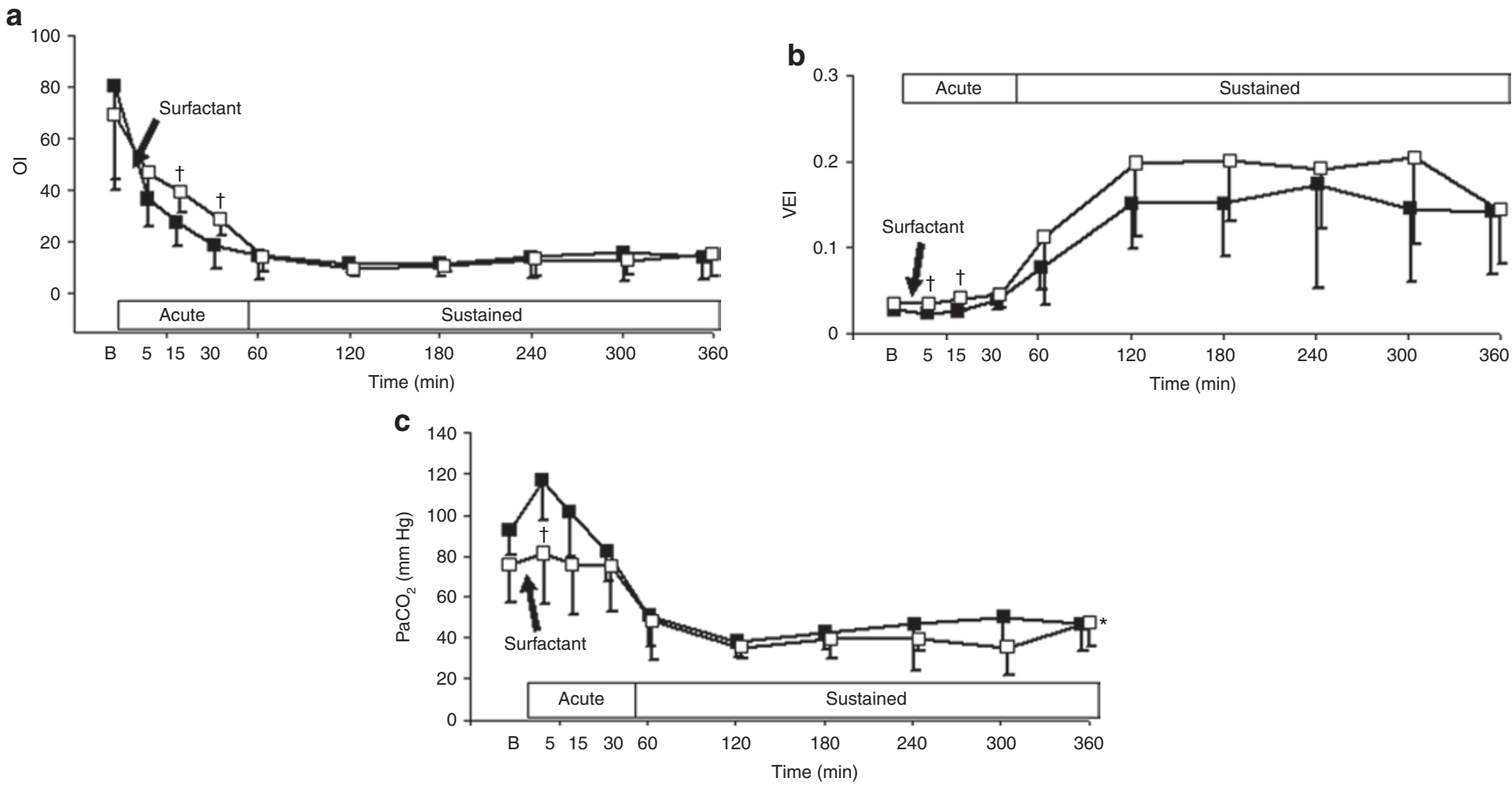

Figure 1. Pulmonary gas exchange and ventilation indexes in premature lambs with RDS treated with SF administered either by bolus (SF-bolus) or aerosolization (SF-aero). (a) Mean oxygenation index, $\mathrm{Ol}$, (b) mean ventilatory efficiency index, VEl, and (c) PaCO, values in the SF-bolus (black squares) and in the SF-aero (white squares) groups. The letter "B" indicates the baseline measurements after stabilization on conventional mechanical ventilation, and the arrow indicates the time of SF administration. Values are mean \pm SD. $* P<0.05$ SF-aero vs. SF-bolus group (two-way ANOVA as function of both group and time); ${ }^{\dagger} P<0.05$ SF-aero vs. SF-bolus group (one-way ANOVA). RDS, respiratory distress syndrome; SF, surfactant. 
a

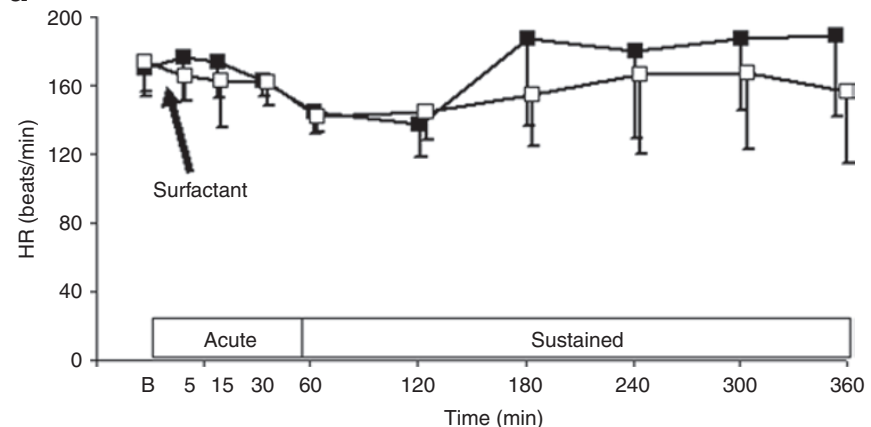

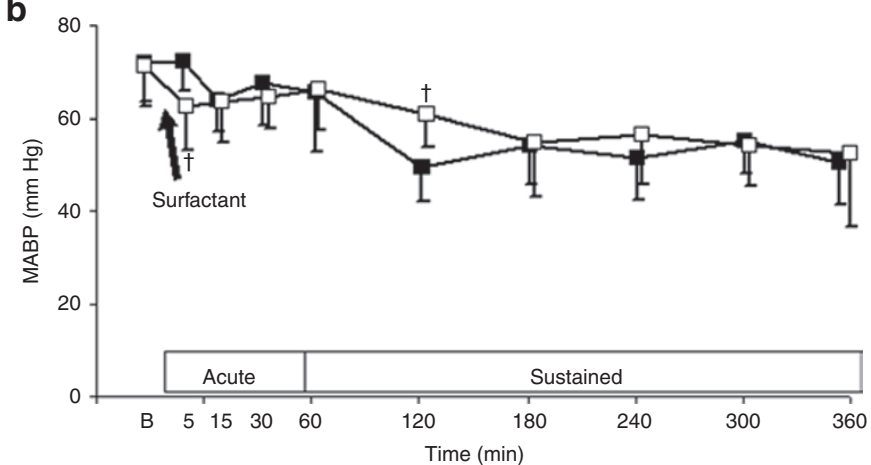

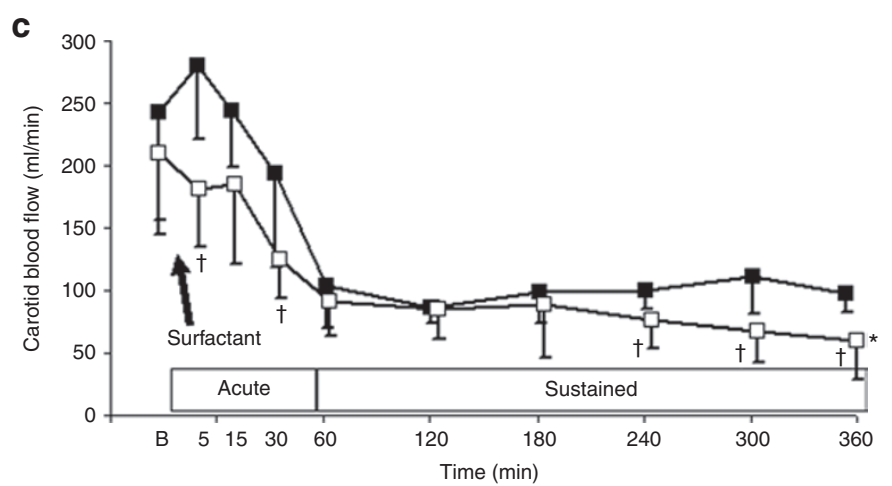

Figure 2. Systemic and cerebral hemodynamics in premature lambs with RDS treated with SF administered either by bolus (SF-bolus) or aerosolization (SF-aero). (a) Mean heart rate (HR), (b) mean arterial blood pressure (MABP), and (c) mean carotid blood flow (CBF) values in the SF-bolus (black squares) and in SF-aero (white squares) groups. The letter " $\mathrm{B}$ " indicates the baseline measurements after stabilization on conventional mechanical ventilation, and the arrow indicates the time of SF administration. Values are mean \pm SD. $* P<0.05$ SF-aero vs. SF-bolus group (two-way ANOVA as function of both group and time); ${ }^{\dagger} P<0.05 \mathrm{SF}$-aero vs. SF-bolus group (one-way ANOVA). RDS, respiratory distress syndrome; SF, surfactant.

a

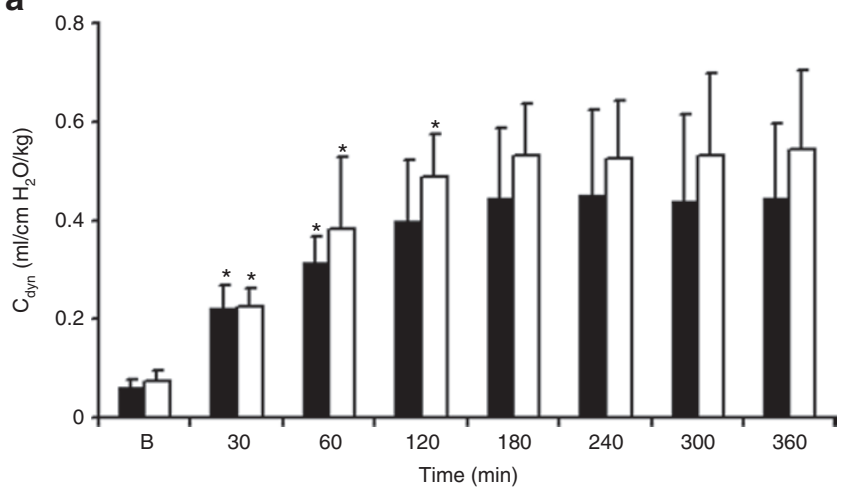

b

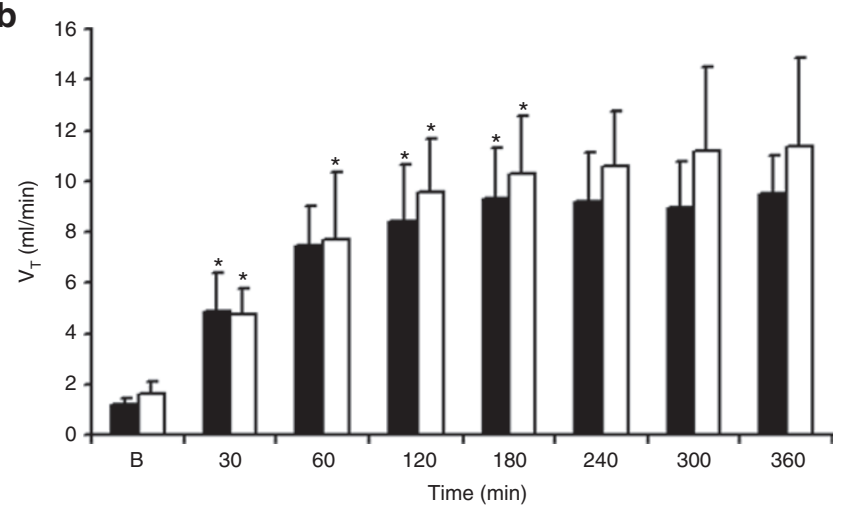

Figure 3. Pulmonary mechanics in premature lambs with RDS treated with SF administered either by bolus (SF-bolus) or aerosolization (SF-aero). (a) Mean dynamic compliance $\left(C_{\text {dyn }}\right)$ and $(\mathbf{b})$ mean tidal volume $\left(V_{T}\right)$ values in the SF-bolus (black bars) and in SF-aero (white bars) groups. The letter " $B^{\prime \prime}$ indicates the baseline measurements after stabilization on conventional mechanical ventilation. Values are mean \pm SD. ${ }^{*} P<0.05$ vs. previous values. RDS, respiratory distress syndrome; SF, surfactant.

throughout the experiment, with no significant differences between the groups (Figure 2b).

Fetal carotid blood flow (CBF) values were also similar in the two groups $(161 \pm 31$ vs. $162 \pm 35 \mathrm{ml} / \mathrm{min}$, NS), as were the baseline values after delivery and stabilization on mechanical ventilation ( $242 \pm 86$ vs. $210 \pm 65 \mathrm{ml} / \mathrm{min}$, NS). Immediately after the SF-bolus administration, a marked increase in CBF was observed, and the flow remained significantly higher than that in the SF-aero group until $30 \mathrm{~min}$ (Figure 2c). By $1 \mathrm{~h}$ after treatment, however, the two groups had similar CBF values. For the rest of the experiment, the $\mathrm{CBF}$ in SF-aero group showed a tendency to decrease slowly, values becoming significantly lower than those measured in the SF-bolus group.

\section{Lung Mechanics}

The mean dynamic compliance at baseline was extremely low in both groups (SF-bolus: $0.06 \pm 0.02 \mathrm{ml} / \mathrm{cm} \mathrm{H}_{2} \mathrm{O} / \mathrm{kg}$ and SF-aero: $0.07 \pm 0.02 \mathrm{ml} / \mathrm{cm} \mathrm{H}_{2} \mathrm{O} / \mathrm{kg}$, NS). After SF treatment, a significant increase was observed in both groups, and by 


\section{Articles | Rey-Santano et al.}
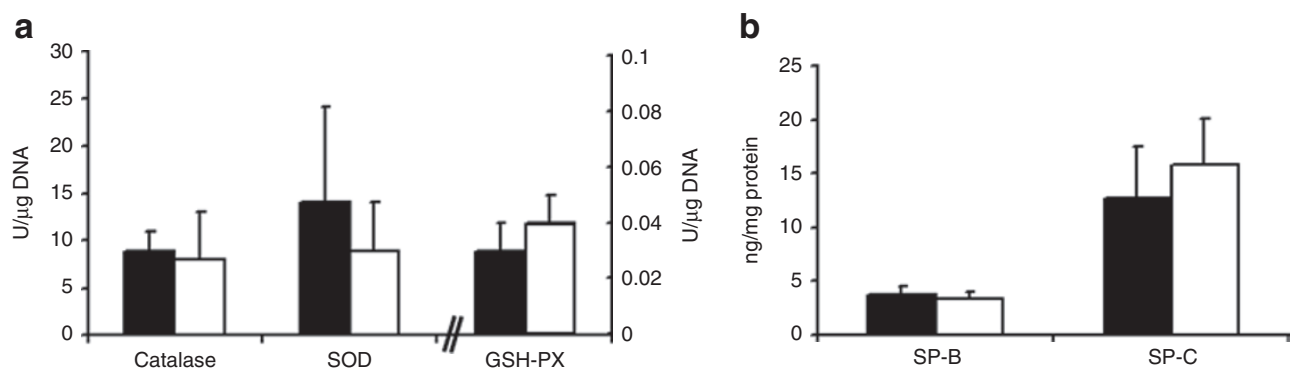

Figure 4. Lung antioxidant enzyme activity and SF protein concentration in premature lambs with RDS treated with SF administered either by bolus (SF-bolus) or aerosolization (SF-aero). (a) Mean lung antioxidant enzyme activity and (b) mean SF protein concentrations in the SF-bolus (black bars) and in SF-aero (white bars) groups. Values are mean \pm SD. Differences between groups were not significant. GSH-PX, glutathione peroxidase; RDS, respiratory distress syndrome; SF, surfactant; SOD, superoxide dismutase; SP, surfactant protein.

a

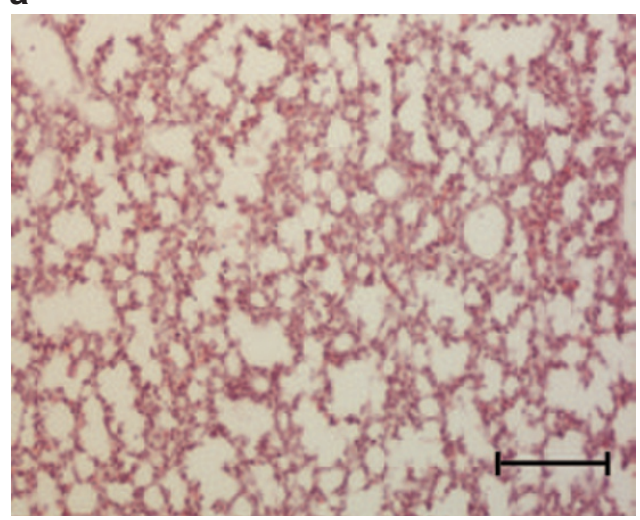

b

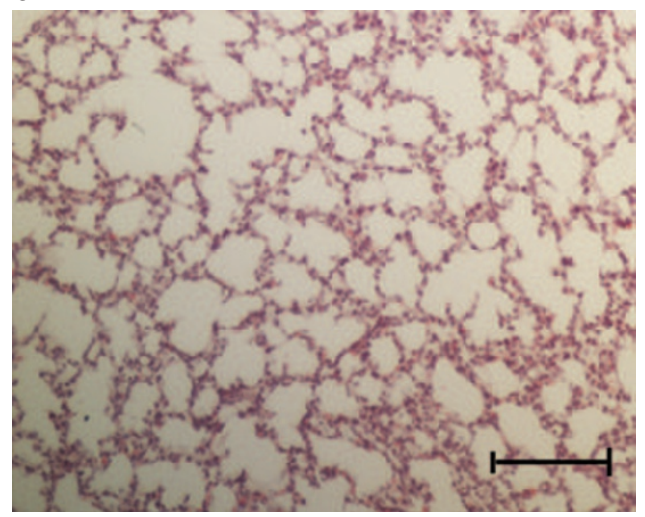

Figure 5. Photomicrographs ( $\times 100$ magnification) of representative lung sections from premature lambs with RDS treated with SF administered either by bolus (SF-bolus) or aerosolization (SF-aero). (a) SF-bolus and (b) SF-aero groups. Panels were obtained from the lower region of the lung. SF, surfactant.

Table 1. Total lung injury scores

\begin{tabular}{|c|c|c|c|c|c|c|c|c|}
\hline & Atelectasis & Necrosis & Edema & $\begin{array}{c}\text { Alveolar } \\
\text { inflammation }\end{array}$ & $\begin{array}{c}\text { Interstitial } \\
\text { inflammation }\end{array}$ & $\begin{array}{c}\text { Alveolar } \\
\text { hemorrhage }\end{array}$ & $\begin{array}{c}\text { Interstitial } \\
\text { hemorrhage }\end{array}$ & Total \\
\hline \multicolumn{9}{|l|}{ Upper } \\
\hline SF-aero & $0.75(0-1)$ & 0 & 0 & $0.37(0-1)^{*}$ & $0.75(0-2)$ & $0.75(0-1)$ & $1.12(1-2)$ & $3.75(3-6)$ \\
\hline \multicolumn{9}{|l|}{ Middle } \\
\hline \multicolumn{9}{|l|}{ Lower } \\
\hline SF-bolus & $2.00(1-3)$ & 0 & 0 & $1.40(1-3)$ & $1.00(0-2)$ & $2.20(1-3)$ & $1.00(1)$ & $7.40(6-9)$ \\
\hline SF-aero & $1.12(0-2)$ & 0 & 0 & $0.62(0-1)$ & $0.75(0-1)$ & $1.12(0-3)^{*}$ & $0.87(0-1)$ & $4.50(2-6)^{*}$ \\
\hline \multicolumn{9}{|l|}{ Total } \\
\hline
\end{tabular}

Statistical differences were assessed using analysis of variance. Values are expressed as mean (range).

SF, surfactant; SF-aero, group treated with SF administered by aerosolization; SF-bolus, group treated with SF administered by bolus.

${ }^{*} P<0.05$ vs. SF-bolus group.

30 min values were significantly higher than baseline $(0.22 \pm$ 0.05 vs. $0.23 \pm 0.04 \mathrm{ml} / \mathrm{cm} \mathrm{H}_{2} \mathrm{O} / \mathrm{kg}$ ). Both groups showed an upward trend for the first $2 \mathrm{~h}$ and then remained stable for the following $3 \mathrm{~h}$ (Figure 3a).
Tidal volume $\left(V_{\mathrm{T}}\right)$ followed a time course similar to that of mean dynamic compliance: it was initially low in both groups (SF-bolus: $1.2 \pm 0.2 \mathrm{ml} / \mathrm{kg}$ vs. SF-aero: $1.6 \pm 0.5 \mathrm{ml} /$ $\mathrm{kg}, \mathrm{NS}$ ), then significantly increased by $30 \mathrm{~min}$ after SF 
administration in both groups $(4.9 \pm 1.5$ vs. $4.8 \pm 1.0 \mathrm{ml} / \mathrm{kg})$ with respect to baseline. $V_{\mathrm{T}}$ increased during the first $3 \mathrm{~h}$ and remained over $10 \mathrm{ml} / \mathrm{kg}$ until the end of the experiment (Figure 3b).

\section{Lung Tissue Analysis}

Figure 4 shows the antioxidant enzyme activity (Figure 4a) and the concentrations of specific SF proteins, surfactant-associated protein (SP)-B and SP-C (Figure $4 \mathrm{~b}$ ). No significant differences were found in catalase, superoxide dismutase, or glutathione peroxidase activity, or in SP-B and SP-C concentrations between the SF-bolus and SF-aero groups. On the other hand, individual injury scores were significantly different for alveolar inflammation and alveolar hemorrhage (Table 1), being higher (corresponding to more severe damage) in the SF-bolus group (Figure $5 a$ ) than in the SF-aero group (Figure $5 b$ ). Furthermore, scores summed for an assessment of total lung injury were also significantly higher in the SF-bolus group (Table 1).

\section{DISCUSSION}

Our results show that in preterm lambs with severe RDS, SF intratracheally delivered as an aerosol improves gas exchange and lung mechanics to the same extent as SF-bolus instillation. Moreover, SF-aero produced less severe alterations in cerebral blood flow and less lung damage as assessed by histological scores than classic bolus instillation.

SF replacement therapy has become standard worldwide for the treatment of neonatal RDS and has significantly reduced the mortality and morbidity of this patient population (14). However, transient hypoxia, hypercapnia, hemodynamic changes including hypotension, CBF fluctuations, and electroencephalogram alterations are commonly observed following the rapid intratracheal instillation of SF $(1,2)$. Moreover, in infants with impaired cerebral autoregulation, hypotension may lead to cerebral hypoperfusion and periventricularintraventricular hemorrhage (15), whereas alterations in CBF and extreme variability in $\mathrm{CBF}$ velocity are major risk factors for brain injury and long-term disability (16).

SF-aero has been proposed as an alternative method of SF administration (11). Eventually, SF-aero administration would not require infants to be intubated or disconnected from ventilatory support, would allow ventilator settings to be fine-tuned (17), and would enhance the pulmonary distribution of the SF (6). However, several factors complicate the aerosol treatment of preterm neonates with exogenous SF (low lung volumes, high respiratory rates, small airways, and high viscosity of SF, among others) (18), so it is understandably difficult to design a successful therapy. Indeed, of the clinical studies performed to date in preterm neonates $(11-13,19)$, only one has shown a moderate improvement in the arterial-alveolar oxygen tension ratio (11), and other studies were inconclusive. On the other hand, the experimental results obtained in various animal models of SF deficiency or depletion vary from SF-aero having hardly any effect $(7,9,10)$ to a dramatic improvement in gas exchange and lung mechanics $(6,8)$. In many of these studies, the amount of material to be aerosolized for a therapeutic response was much higher than the doses used in SF-bolus administration, and the therapeutic response was lower during aerosolization $(6,7,9)$.

It is noteworthy that in the current study a standard SF dose for established RDS (20) was used in both groups, and equivalent responses were observed in terms of gas exchange, ventilation indexes, and lung function. Moreover, because during aerosolization the smaller aerosol particles may be exhaled, it can be assumed that the SF dose that effectively reached the lungs was less than the nominal dose $(200 \mathrm{mg} / \mathrm{kg})$. Unfortunately, it was not possible to determine the amount of exhaled SF in this work, but according to our bench studies and considering the study conditions (e.g., temperature, humidity), SF aerosol loss can be estimated to have been $\sim 20-30 \%$ (21).

We observed some differences in the acute response to SF administration. The rapid SF-bolus administration produced an immediate decrease in the OI in contrast to the gradual decrease observed after the SF aerosolization. This slower response of the OI with aerosolization does not need to be seen as a negative result because a sudden increase in $\mathrm{PaO}_{2}$ might be associated with a quick fall in pulmonary vascular resistance, secondary to an improvement in lung expansion and gas exchange induced by instillation of SF. Following SF-bolus treatment, pulmonary hemorrhage and inflammation, resulting from stress failure of pulmonary capillaries, have been associated with a rapid fall in pulmonary vascular resistance and an increase in left-to-right shunting though a patent ductus arteriosus $(22,23)$. In our study, in agreement with those findings, the SF-bolus group had a significantly higher score for alveolar hemorrhage and inflammation. The gradual administration of SF during aerosolization seems to have reduced the acute SF load on the upper airways, avoiding hypercapnia and allowing the newly deposited SF to spread until the next incoming SF jet arrived in the next respiratory cycle. Therefore, every SF jet might have contributed to the stepwise recruitment of lung units, improving both gas exchange and lung mechanics, which would explain why the OI changes were more gradual.

Although rapid systemic and cerebral hemodynamic changes have been described in preterm infants immediately after the start of the therapy, associated with rapid changes in oxygenation, carbon dioxide, and/or lung function $(1,2)$, little is known about the effects of SF-aero on systemic and cerebral hemodynamics. Dijk et al. (9) demonstrated in an adult model of acute lung injury that SF-aero may partly avoid the adverse effects of bolus instillation, hemodynamic stability, and less severe changes in CBF being observed in comparison with the significant drop in blood pressure and $\mathrm{CBF}$ following rapid bolus instillation. Our results are consistent with these findings in that we observed no change in blood pressure and a gradual decrease in cerebral blood flow in the preterm lambs that received SF-aero. On the other hand, in the study by Dijk et al. (9), SF-bolus administration did not produce hypercapnia, and a significant decrease in CBF drop was reported. Our preterm lambs developed hypercapnia and showed an increase in $\mathrm{CBF}$ with no change 
in blood pressure immediately after the rapid bolus instillation. This might be explained by the different animal models used in each study (adult rabbits vs. preterm lambs), specifically the immaturity of the cardiovascular system in preterm lambs. It is known there are substantial structural and functional differences between the mature cardiovascular system (adult rabbit) and that of the premature cardiovascular system (premature lamb), particularly during the early period of transition to extrauterine life (e.g., ductus arteriosus, foramen ovale, premature myocardium, autonomic nervous system) (24). Therefore, it seems reasonable to suppose that the SF instillation in preterm lambs more accurately reflects the sequence of events that occurs in preterm infants because hypercapnia and CBF increases following SF therapy have often been reported in very preterm infants linked to a transient airway obstruction by instilled SF $(15,25)$. Therefore, a transient airway obstruction following bolus instillation would have produced high $\mathrm{CO}_{2}$ levels and led to an increase in CBF (25). Because brain injury has been associated with CBF fluctuations, a new therapeutic approach based on SF-aero could represent an important clinical advance.

Various alternatives to SF-bolus administration have been proposed to avoid or reduce the adverse effects on the cerebral circulation $(4,5)$. Although it is currently assumed that the optimal effect will occur when SF is distributed homogeneously throughout the lung, different bolus administration procedures can affect subsequent SF distribution and efficacy. Differences in the uniformity of distribution after slow infusion (during $45 \mathrm{~min}$ ) as compared with bolus instillation have been observed (4). Furthermore, instillation of four fractional SF doses did not result in the same enhancement of gas exchange as the same dose given in a single bolus (5).

SF-bolus administration modulates the pulmonary inflammatory cascade, inhibiting oxidative burst activity (26), and stimulates endogenous SF synthesis $(27,28)$. We tested the hypothesis that if SF delivered as an aerosol achieves a similar response in terms of gas exchange and lung mechanics, the antioxidant enzyme activity would at least correspond to that observed after SF-bolus administration. In our study, SF-aero showed a trend toward lower catalase and superoxide dismutase concentrations and equivalently high glutathione peroxidase values, but the differences were not statistically significant, probably due to the sample size and the limited follow-up period. Moreover, our results suggest that SF administration promotes endogenous SF synthesis, showing specific SP-B and SP-C concentrations in lung tissue homogenates, values being significantly higher than those in non-SF-treated animals (data not shown). Moreover, an upward trend in SP-C concentration was observed in animals given SF-aero in comparison with those treated with bolus instillation. Perhaps better SF distribution in the SF-aero group could have produced an upregulation in the synthesis of endogenous SF as compared with that in the SF-bolus group; this would be in line with the trend toward greater compliance and the significant sustained increase in compliance observed at $2 \mathrm{~h}$ in the aerosolized group but not in the animals treated with SF-bolus.
Unfortunately, lung distribution of SF could not be measured due to technical limitations associated with aerosolization. In an attempt to determine the penetration of SF into the lungs, histological assessments of the upper, middle, and lower lobes were performed by an expert pathologist blinded to the experimental design. The histological findings indicate that overall, SF-aero reduced the total lung damage and suggest a better SF distribution within the lungs; in particular, SF was administered in the supine position in both groups, but a significantly higher histological score was obtained in the distal lung in lambs treated with SF-aero.

In summary, our study shows for the first time that a standard dose of SF for established RDS delivered as an aerosol can produce a similar response to rapid intratracheal bolus instillation of the same dose, in terms of gas exchange and pulmonary mechanics, and result in less lung damage. Moreover, SF aerosolization reduced the initial hypercapnia seen in animals treated with SF-bolus and produced a more gradual and stable pattern in cerebral hemodynamics. Although these results are encouraging, it must be taken into account that a primary goal of SF-aero is to avoid intratracheal intubation, and in the current study, an inhalation catheter was used to produce intratracheal SF aerosols with animals paralyzed, sedated, and under cardiovascular management. Future studies focusing on administering SF-aero during noninvasive ventilatory support are needed.

\section{METHODS}

The experimental protocol complied with all current regulations on animal research (EU86/609, RD 1201/2005, and those of the Experimental Research Committee of the Cruces University Hospital). This comparative study was carried out using 14 premature Latxa lambs delivered at $133 \mathrm{~d}$ of gestation (term $=145 \mathrm{~d}$ ), it having been shown that there is severe pulmonary SF deficiency in lambs of this breed at this gestational age $(29,30)$. Mean body weight of the lambs at birth was $3.1 \pm 0.4 \mathrm{~kg}$.

\section{Animal Preparation}

Ewes were sedated with xylazine $(0.15 \mathrm{mg} / \mathrm{kg}$ i.m. $)$ and ketamine $(5 \mathrm{mg} / \mathrm{kg}$ i.v.), and anesthesia was maintained with propofol $(30-40 \mathrm{mg} / \mathrm{kg} / \mathrm{h})$. A tracheal tube (internal diameter of $8 \mathrm{~mm}$ ) was inserted and connected to a volume-controlled ventilator (Julian; Dräger, Lübeck, Germany). The initial settings were respiratory frequency of 30 cycles per min; peak inspiratory pressure (PIP)/positive end-expiratory pressure: $14-16 / 2 \mathrm{~cm} \mathrm{H}_{2} \mathrm{O}$; inspiration/expiration ratio: 1:2; and $\mathrm{FiO}_{2}: 0.4-0.6$ to maintain normal levels of $\mathrm{O}_{2}$ and $\mathrm{CO}_{2}$. A peripheral arterial cannula was inserted to monitor blood gases, MABP, and heart rate. The uterus was exposed by a subcostal incision, and the head and neck of the fetal lambs exteriorized. For each lamb, a 4.0-mm-internal diameter tracheal tube (Hi-LoJet; Mallinckrodt, St Louis, MO) was inserted by tracheotomy and tied to prevent leaks, and then lungs were allowed to slowly drain by gravity. Catheters were placed in the jugular vein to maintain fluid balance and anesthesia and in the axillary artery to obtain arterial blood for gas analysis and to measure MABP and heart rate. The carotid artery was isolated, and a noninvasive perivascular Doppler flow probe (MA3PS; Transonic Systems, Ithaca, FL) was used to continuously measure CBF. Lambs were given i.v. ketamine ( $3 \mathrm{mg} /$ $\mathrm{kg})$, fentanyl $(5 \mu \mathrm{g} / \mathrm{kg})$, and atracurium besylate $(0.1 \mathrm{mg} / \mathrm{kg})$, and the umbilical cord was cut.

Lambs were weighed and connected to a neonatal ventilator (BP-200; Bear Medical Systems, Riverside, CA). The initial settings were: respiratory frequency: 60 cycles per min; PIP/positive 
end-expiratory pressure: $30 / 8 \mathrm{~cm} \mathrm{H}_{2} \mathrm{O}$; inspiration/expiration ratio: $1: 2 ; \mathrm{FiO}_{2}$ : 1.0 ; and flow: $10 \mathrm{l} / \mathrm{min}$. Subsequently, the PIP, respiratory frequency, and $\mathrm{FiO}_{2}$ were varied with the goal of keeping $\mathrm{PaCO}_{2}$ values within a normal range of $35-45 \mathrm{~mm} \mathrm{Hg}$ and $\mathrm{PaO}_{2}$ between

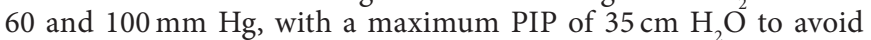
pneumothorax. Ketamine $(5 \mathrm{mg} / \mathrm{kg} / \mathrm{h})$ and fentanyl $(4 \mu \mathrm{g} / \mathrm{kg} / \mathrm{min})$ were infused to maintain anesthesia and analgesia, and atracurium besylate $(1.2 \mathrm{mg} / \mathrm{kg})$ was infused to prevent spontaneous breathing. A MABP of $40 \mathrm{~mm} \mathrm{Hg}$ was maintained by infusing dopamine at $\sim 5-10 \mu \mathrm{g} / \mathrm{kg} / \mathrm{min}$ as needed.

\section{Experimental Design}

Premature lambs were stabilized on the ventilator before any treatment was given and were randomly assigned to one of the two groups.

SF-bolus Group. These animals received $200 \mathrm{mg} / \mathrm{kg}$ of poractant alfa (Curosurf; Chiesi Farmaceutici, Parma, Italy) in a supine position; this was administered without disconnecting the animal from the ventilator $(n=7)$.

SF-aero Group. These animals also received $200 \mathrm{mg} / \mathrm{kg}$ of poractant alfa continuously aerosolized (during inspiration) over a period of 20 min $(n=7)$, delivered intratracheally by means of inhalation catheters (AeroProbe; Trudell Medical, London, Ontario, Canada) connected to a pneumatic compressor (LABneb ${ }^{\star} \mathrm{CCS}$; Trudell Medical) (30). This aerosol system can deliver up to $0.4 \mathrm{ml}$ of SF per min during mechanical ventilation, with $80 \%$ of the particles having a diameter of $<5 \mu \mathrm{m}(21,31)$.

\section{Lung Mechanics}

Lung mechanics were measured with a computerized system (PowerLab/16SP and Chart5; ADInstruments, Colorado Springs, CO) (32). Airflow was measured by a pneumotachometer (Fleisch 00; OEM Medical, Richmond, VA), and pressure was measured with a differential pressure transducer (MP45; Validyne Engineering, Northridge, CA). Volume was then calculated by integration of the flow signal. The computer system uses the least mean square analysis technique to determine the pulmonary mechanics (33). Ten random breaths were analyzed for each time-point interval. The analyzer reported values for mean dynamic compliance and $V_{\mathrm{T}}$.

\section{Measurements}

Arterial $\mathrm{pH}, \mathrm{PaO}_{2}$, and $\mathrm{PaCO}_{2}$ (GemPremier 4000; Instrumentation Laboratory, Lexington, MA) and systemic and cerebral hemodynamic parameters (MABP, heart rate, and CBF) were continuously recorded (OmniCare GMS 24; Hewlett Packard, Boeblingen, Germany).

The OI was calculated as mean airway pressure $\left(\mathrm{cm} \mathrm{H}_{2} \mathrm{O}\right) \times \mathrm{FiO}_{2} /$ $\mathrm{PaO}_{2}(\mathrm{~mm} \mathrm{Hg}) \times 100(34)$. To assess pulmonary ventilation independent of ventilator settings, ventilatory efficiency index was calculated as $3,800 / \mathrm{PIP}$ - positive end-expiratory pressure $\left(\mathrm{cm} \mathrm{H}_{2} \mathrm{O}\right) \times$ respiratory frequency (cycles per min) $\times \mathrm{PaCO}_{2}(34)$.

Gas exchange and hemodynamic parameters were recorded during fetal life (fetal), after connection to the ventilator and just before SF administration (baseline) and again 5, 15, and 30 min later to assess the acute effect of SF administration. Subsequently, these parameters were recorded every $30 \mathrm{~min}$, for the rest of the experiment, to monitor the longer-term effects of the therapy. Lung mechanics and ventilatory indexes were measured at baseline and again every $30 \mathrm{~min}$ until the end of the experiment, at $6 \mathrm{~h}$.

\section{Lung Tissue Analysis}

At postmortem, the lungs were removed and perfused with saline. The left lung bronchus was isolated, occluded, placed into dry ice, and stored at $-80^{\circ} \mathrm{C}$ until used for the biochemical assays (35), whereas the right lung was fixed in $4 \%$ formaldehyde at $15 \mathrm{~cm} \mathrm{H}_{2} \mathrm{O}$ for histological analysis.

\section{Antioxidant Enzyme Activity}

Frozen lung samples were weighed $(100 \mathrm{mg})$, homogenized with $0.4 \mathrm{ml}$ of ice-cold phosphate buffer $(50 \mathrm{mmol} / \mathrm{l}, \mathrm{pH} 7.4)$ containing $1 \mathrm{mmol} / \mathrm{l} \mathrm{EDTA}$, centrifuged $\left(15,000 \mathrm{~g}, 10 \mathrm{~min}, 4^{\circ} \mathrm{C}\right)$, and the supernatant removed (36). Catalase activity assays were used to measure the reduction of $\mathrm{H}_{2} \mathrm{O}_{2}$ to $\mathrm{H}_{2} \mathrm{O}$ by catalase (37), in which one unit of catalase is defined as the amount of enzyme needed to react with $1 \mu \mathrm{mol}$ of $\mathrm{H}_{2} \mathrm{O}_{2}$ per min. Similarly, glutathione peroxidase activity assays were used to measure the reduction of $\mathrm{H}_{2} \mathrm{O}_{2}$ to $\mathrm{H}_{2} \mathrm{O}$ by glutathione peroxidase (38), with one unit of glutathione peroxidase activity being defined as the oxidation of $1 \mu \mathrm{mol}$ of nicotinamide adenine dinucleotide phosphate per min. Furthermore, we performed superoxide dismutase assays (Cayman Chemical, Ann Arbor, MI), and one unit of superoxide dismutase was defined as the amount of enzyme needed to exhibit 50\% dismutation of the superoxide radical. Finally, DNA measurements were performed using a fluorometric method that relies on the intercalation of a fluorescent dye (Hoechst 33342; Sigma-Aldrich, St Louis, MO) into intact double-stranded DNA (39). Results for antioxidant enzymes are expressed as $\mathrm{U} / \mu \mathrm{g}$ of DNA.

$S P-B$ and SP-C Concentrations. Frozen lung tissues $(0.2 \mathrm{~g})$ were homogenized in $1 \mathrm{ml}$ of Tissue Protein Extraction Reagent (T-PER; Thermo-Scientific, Rockford, IL) containing Complete Protease Inhibitor Cocktail (Sigma-Aldrich). The tissue homogenates were centrifuged at $12,000 \mathrm{~g}$ for $10 \mathrm{~min}$ at $4^{\circ} \mathrm{C}$, and supernatant (protein extract) was collected. Samples were then added to a microtiter plate with wells containing a biotin-conjugated antibody preparation specific for ovine SP-B or SP-C (Cusabio; Wuhan, Hubei Province, China). The protein concentration was then determined spectrophotometrically at $450 \mathrm{~nm}$ (POLARstar; BMG, Ortenberg, Germany). The Bradford protein assay (Bio-Rad, Hercules, CA) was used for determining the concentration of protein in solution. Results for SP-B and SP-C are expressed as ng/ mg of protein.

Histological Score. Samples of the right lung lobes were embedded in paraffin wax, and 5- $\mu \mathrm{m}$ sections were obtained from samples of the upper, middle, and lower lobes as follows: one sample from the cranial-ventral (nondependent) upper lobe, one from the caudal-dorsal (dependent) upper lobe, two from the middle lobe, and another two from the lower lobe. Slides from each section were stained with hematoxylin and eosin and analyzed qualitatively by light microscopy. Scoring of lung injury was performed by a pathologist blinded to the experimental design using a semiquantitative scoring system. Pathological signs of lung injury, namely, alveolar and interstitial inflammation, alveolar and interstitial hemorrhage, edema, atelectasis, and necrosis, were each scored on a 0-4-point scale: 0: no injury; 1: injury to $25 \%$ of the field; 2 : injury to $50 \%$ of the field; 3 : injury to $75 \%$ of the field; and 4 : injury across the field (40).

\section{Statistical Analysis}

Values are expressed as mean \pm SD. Data were analyzed with the Levene test to confirm the homogeneity of variance between the different treatments and with the Kolmogorov-Smirnov test for normality (JMP8 Statistical Discovery; SAS, Cary, NC). One-way ANOVA was performed to assess whether gas exchange, systemic and cerebral hemodynamic parameters, ventilation indexes, SF protein concentrations, and antioxidant enzyme activity varied as a function of group. Comparisons of results (gas exchange, hemodynamic parameters, and ventilatory indexes) were performed by repeated-measures two-way ANOVA as a function of group and time. Lung injury scores and lung mechanics were analyzed using the Kruskal-Wallis test and Mann-Whitney $U$ test, as appropriate. A $P$ value of 0.05 was accepted as significant.

\section{ACKNOWLEDGMENTS}

The excellent technical assistance of Francisco Jose Alvarez-Diaz and Hector Lafuente is gratefully acknowledged.

\section{STATEMENT OF FINANCIAL SUPPORT}

This study was partially supported by grants from the Spanish Carlos III Health Institute: FIS 10/943 and RD08/072 (Red SAMID: Maternal, Child Health and Development Research Network, within the framework of the VI Spanish National Plan for R+D+i 2008-2012) and the Government of the Basque Country (grant 2007111046).

Disclosure: The authors declare no conflict of interest. 


\section{REFERENCES}

1. Schipper JA, Mohammad GI, van Straaten HL, Koppe JG. The impact of surfactant replacement therapy on cerebral and systemic circulation and lung function. Eur J Pediatr 1997;156:224-7.

2. Murdoch E, Kempley ST. Randomized trial examining cerebral haemodynamics following artificial or animal surfactant. Acta Paediatr 1998;87:411-5.

3. Fujii AM, Bailey J, Doros G, et al. Effects of beractant and poractant administration on cerebral hemodynamics. J Neonatal-Perinat Med 2009;2:27-34.

4. Segerer H, van Gelder W, Angenent FW,et al. Pulmonary distribution and efficacy of exogenous surfactant in lung-lavaged rabbits are influenced by the instillation technique. Pediatr Res 1993;34:490-4.

5. Alvarez FJ, Alfonso LF, Gastiasoro E, Lopez-Heredia J, Arnaiz A, Vallsi-Soler A. The effects of multiple small doses of exogenous surfactant on experimental respiratory failure induced by lung lavage in rats. Acta Anaesthesiol Scand 1995;39:970-4.

6. Lewis JF, Ikegami M, Jobe AH, Tabor B. Aerosolized surfactant treatment of preterm lambs. J Appl Physiol 1991;70:869-76.

7. Henry MD, Rebello CM, Ikegami M, Jobe AH, Langenback EG, Davis JM. Ultrasonic nebulized in comparison with instilled surfactant treatment of preterm lambs. Am J Respir Crit Care Med 1996;154(2 Pt 1):366-75.

8. Ellyett KM, Broadbent RS, Fawcett ER, Campbell AJ. Surfactant aerosol treatment of respiratory distress syndrome in the spontaneously breathing premature rabbit. Pediatr Res 1996;39:953-7.

9. Dijk PH, Heikamp A, Bambang Oetomo S. Surfactant nebulisation prevents the adverse effects of surfactant therapy on blood pressure and cerebral blood flow in rabbits with severe respiratory failure. Intensive Care Med 1997;23:1077-81.

10. Fok TF, al-Essa M, Dolovich M, Rasid F, Kirpalani H. Nebulisation of surfactants in an animal model of neonatal respiratory distress. Arch Dis Child Fetal Neonatal Ed 1998;78:F3-9.

11. Jorch G, Hartl H, Roth B, et al. Surfactant aerosol treatment of respiratory distress syndrome in spontaneously breathing premature infants. Pediatr Pulmonol 1997;24:222-4.

12. Berggren E, Liljedahl M, Winbladh B, et al. Pilot study of nebulized surfactant therapy for neonatal respiratory distress syndrome. Acta Paediatr 2000;89:460-4.

13. Finer NN, Merritt TA, Bernstein G, Job L, Mazela J, Segal R. An open label, pilot study of Aerosurf ${ }^{ø}$ combined with nCPAP to prevent RDS in preterm neonates. J Aerosol Med Pulm Drug Deliv 2010;23:303-9.

14. Schwartz RM, Luby AM, Scanlon JW, Kellogg RJ. Effect of surfactant on morbidity, mortality, and resource use in newborn infants weighing 500 to 1500 g. N Engl J Med 1994;330:1476-80.

15. Kaiser JR, Gauss CH, Williams DK. Surfactant administration acutely affects cerebral and systemic hemodynamics and gas exchange in verylow-birth-weight infants. J Pediatr 2004;144:809-14.

16. Ballabh P. Intraventricular hemorrhage in premature infants: mechanism of disease. Pediatr Res 2010;67:1-8.

17. Ruppert C, Kuchenbuch T, Boensch M, et al. Dry powder aerosolization of a recombinant surfactant protein-C-based surfactant for inhalative treatment of the acutely inflamed lung. Crit Care Med 2010;38: 1584-91.

18. Lu KW, Pérez-Gil J, Taeusch H. Kinematic viscosity of therapeutic pulmonary surfactants with added polymers. Biochim Biophys Acta 2009;1788:632-7.

19. Arroe M, Pedersen-Bjergaard L, Alberstsen P, et al. Inhalation of aerosolized surfactant (Exosurf) to neonates treated with nasal continuous positive airway pressure. Prenat Neonat Med 1998;3:346-52.

20. Speer CP, Gefeller O, Groneck P, et al. Randomised clinical trial of two treatment regimens of natural surfactant preparations in neonatal respiratory distress syndrome. Arch Dis Child Fetal Neonatal Ed 1995;72:F8-13.
21. Murgia X, Gastiasoro E, Mielgo V, et al. Surfactant and perfluorocarbon aerosolization during different mechanical ventilation strategies by means of inhalation catheters: an in vitro study. J Aerosol Med Pulm Drug Deliv 2012;25:23-31.

22. Kumar A, Lakkundi A, McNamara PJ, Sehgal A. Surfactant and patent ductus arteriosus. Indian J Pediatr 2010;77:51-5.

23. Merritt TA, Hallman M, Bloom BT, et al. Prophylactic treatment of very premature infants with human surfactant. N Engl J Med 1986;315: 785-90.

24. Shukla AC, Steven JM, McGowan FX. Cardiac physiology and pharmacology. In: Cote CJ, Lerman J, Todres P, eds. A Practice of Anesthesia for Infants and Children. Philadelphia: Saunders, 2009:361-96.

25. Tarawneh A, Kaczmarek J, Bottino MN, Santanna GM. Severe airway obstruction during surfactant administration using a standardized protocol: a prospective, observational study. J Perinatol 2012;32:270-5.

26. Dizdar EA, Uras N, Oguz S, et al. Total antioxidant capacity and total oxidant status after surfactant treatment in preterm infants with respiratory distress syndrome. Ann Clin Biochem 2011;48(Pt 5):462-7.

27. Bunt JE, Carnielli VP, Janssen DJ, et al. Treatment with exogenous surfactant stimulates endogenous surfactant synthesis in premature infants with respiratory distress syndrome. Crit Care Med 2000;28:3383-8.

28. Ikegami M, Jobe A, Yamada T, et al. Surfactant metabolism in surfactanttreated preterm ventilated lambs. J Appl Physiol 1989;67:429-37.

29. Gastiasoro-Cuesta E, Alvarez-Diaz FJ, Rey-Santano C, Arnaiz-Renedo A, Loureiro-Gonzalez B, Valls-i-Soler A. Acute and sustained effects of lucinactant versus poractant-alpha on pulmonary gas exchange and mechanics in premature lambs with respiratory distress syndrome. Pediatrics 2006;117:295-303.

30. Murgia X, Mielgo V, Valls-i-Soler A, Ruiz-del-Yerro E, Rey-Santano C. Aerosolized perfluorocarbon improves gas exchange and pulmonary mechanics in preterm lambs with severe respiratory distress syndrome. Pediatr Res 2012;72:393-9.

31. Murgia X, Gastiasoro E, Mielgo V, et al. Surfactant and perfluorocarbon aerosolization by means of inhalation catheters for the treatment of respiratory distress syndrome: an in vitro study. J Aerosol Med Pulm Drug Deliv 2011;24:81-7.

32. Alvarez FJ, Gastiasoro E, Rey-Santano MC, Gomez-Solaetxe MA, Publicover NG, Larrabe JL. Dynamic and quasi-static lung mechanics system for gas-assisted and liquid-assisted ventilation. IEEE Trans Biomed Eng 2009;56:1938-48.

33. Bhutani VK, Sivieri EM, Abbasi S, Shaffer TH. Evaluation of neonatal pulmonary mechanics and energetics: a two factor least mean square analysis. Pediatr Pulmonol 1988;4:150-8.

34. Rey-Santano C, Alvarez-Diaz FJ, Mielgo V, et al. Bronchoalveolar lavage versus bolus administration of lucinactant, a synthetic surfactant in meconium aspiration in newborn lambs. Pediatr Pulmonol 2011;46:991-9.

35. Ikeda T, Murata Y, Quilligan EJ, Parer JT, Murayama T, Koono M. Histologic and biochemical study of the brain, heart, kidney, and liver in asphyxia caused by occlusion of the umbilical cord in near-term fetal lambs. Am J Obstet Gynecol 2000;182:449-57.

36. Rey-Santano C, Mielgo VE, Gastiasoro E, et al. Early cerebral hemodynamic, metabolic, and histological changes in hypoxic-ischemic fetal lambs during postnatal life. Front Neurosci 2011;5:111.

37. Holmes RS, Masters CJ. On the tissue and subcellular distribution of multiple forms of catalase in the rat. Biochim Biophys Acta 1969;191:488-90.

38. Paglia DE, Valentine WN. Studies on the quantitative and qualitative characterization of erythrocyte glutathione peroxidase. J Lab Clin Med 1967;70:158-69.

39. Labarca C, Paigen K. A simple, rapid, and sensitive DNA assay procedure. Anal Biochem 1980;102:344-52.

40. Zimmermann AM, Roberts KD, Lampland AL, et al. Improved gas exchange and survival after KL-4 surfactant in newborn pigs with severe acute lung injury. Pediatr Pulmonol 2010;45:782-8. 